\title{
Helicobacter pylori: aspetti di sanità pubblica
}

\section{Pietro Ragni,}

Direzione Sanitaria Azienda USL di Reggio Emilia; Assessorato alla Sanità della

Regione Emilia-Romagna, Servizio Sanità Pubblica

\section{RELAZIONE}

Il riconoscimento del ruolo eziologico e patogenetico di Helicobacter pylori $(\mathrm{Hp})$ nei confronti del cancro gastrico, nonché i progressi diagnostici e i successi terapeutici nell'eradicazione del germe ci pongono il quesito relativo all'opportunità di uno screening di massa di tale infezione. Come è noto, la questione è già stata affrontata in più sedi e in più occasioni, con risposta pressoché costantemente negativa.

Scopo di questo lavoro è rivedere le motivazioni che stanno alla base delle attuali raccomandazioni, ripercorrendo dapprima ai criteri necessari per proporre un test ad una ampia fetta di popolazione, ed esaminando poi rispetto ad essi la situazione specifica dell'infezione da Hp.

Innanzitutto, occorre chiarire che cosa si intende per screening, dato che la definizione tecnica è diversa da ciò che spesso si pensa.

Lo screening è un procedimento di selezione che si propone di identificare gli individui che corrono un rischio sufficientemente alto di malattia, per garantire un'ulteriore ricerca o un'azione preventiva diretta. La ricerca viene sistematicamente offerta ad una popolazione di individui che non hanno richiesto l'intervento medico (1).

Lo screening non deve quindi essere confuso con altre strategie di ricerca diagnostica, in particolar modo con il "case-finding", cioè la ricerca di una patologia in persone ritenute ad alto rischio che per un motivo qualsiasi si presentino all'osservazione medica; a maggior ragione non deve essere confuso con l'approfondimento diagnostico su persone sintomatiche.

\section{I principi dello screening}

Esistono criteri ormai ben codificati per valutare l'opportunità di sottoporre a screening una vasta fetta di popolazione (2). Sono criteri ampiamente condivisi nel mondo scientifico e fatti propri dall'Organizzazione Mondiale della Sanità

In particolare, per sottoporre a un dato screening la popolazione, occorre che:

1. La malattia costituisca una minaccia grave per la salute pubblica;

2. Ai soggetti nei quali la malattia è stata scoperta possa essere applicato un trattamento di efficacia dimostrata;

3. Si disponga di mezzi adeguati di diagnosi e di trattamento;

4. Vi sia evidenza di un vantaggioso rapporto costo/efficacia sia come guadagno di salute a lungo termine, sia di risorse risparmiate con la prevenzione;

5. La malattia possa essere scoperta in fase di latenza o all'inizio della fase clinica;

6. Esista un test o un esame che permetta di rintracciare effettivamente la malattia;

7. Il test utilizzato sia accettabile dalla popolazione;

8. Conoscere bene la storia naturale della malattia, specialmente nella sua evoluzione dalla fase di latenza alla fase sintomatica;

9. I soggetti che riceveranno un trattamento siano scelti attraverso criteri conosciuti in precedenza;

10.Il costo della ricerca dei casi (comprese spese di diagnostica e di trattamento dei soggetti riconosciuti malati) non sia sproporzionata rispetto al costo globale delle cure mediche.

Proviamo ora a scorrere i punti principali e dare loro risposta.

La malattia costituisce una minaccia grave per la salute pubblica?

L'infezione è endemica in tutto il mondo, ma con forte eterogeneità geografica. Nei Paesi in via di sviluppo la prevalenza risulta spesso superiore all' $80 \%$, mentre nei Paesi industrializzati si hanno valori oscillanti fra il 20 e il $50 \%$ (3). In entrambi i casi si hanno pi forti differenze fra ceti socioeconomici, con aumento della prevalenza fra gli strati più poveri e le famiglie più numerose.

Hp è ritenuto la causa di diverse patologie quali: ulcera duodenale o gastrica, atrofia mucosa carcinoma gastrico linfoma gastrico (4). Tuttavia, l'impatto più rilevante sulla salute pubblica è dovuto alla malattia più grave causata da $\mathrm{Hp}$, che è il tumore maligno dello stomaco.

Si tratta di un tumore la cui diffusione è in declino da alcune decadi, e tuttavia ancora epidemiologicamente molto rilevante. "Gastric cancer is a major public health issue" è una delle affermazioni indiscusse del Consensus Report di Maastricht del febbraio 2002 (European Helycobacter Pylori Study Group) $(5,6)$.

L'incidenza in Italia nel periodo 1990-92 era di circa 35/100.000 negli uomini e di 20-25/100.000 
nelle donne, con punte fino a 57/100.000 negli uomini in Romagna. La mortalità in EmiliaRomagna nel 1997 era di 28/100.000 nei maschi e di 20/100.000 nelle femmine.

Ai soggetti nei quali la malattia è stata scoperta possa essere applicato un trattamento la cui efficacia sia stata dimostrata?

L'efficacia del trattamento nell'eradicazione di Hp è molto alta: a seconda degli schemi utilizzati, e considerando sia i trattamenti di prima che di seconda linea, sono riportati successi in percentuali che vanno dall' 80 al 98\% (7).

L'efficacia del trattamento si manifesta verosimilmente anche nel ridurre la probabilità di sviluppare il cancro dello stomaco. In uno studio giapponese eseguito su 1526 soggetti, il tumore gastrico si sviluppò nel $2.9 \%$ di 1246 pazienti con Hp nell'arco di 7.8 anni, mentre nessun caso veniva osservato nei 280 soggetti di controllo non infetti. Più importante ancora è che nessun caso di cancro veniva osservato in un sottogruppo di 253 pazienti precedentemente infetti e sottoposti a trattamento eradicante precoce nel follow-up (8).

Tuttavia, le problematiche relative al trattamento non si esauriscono con questi dati, e addirittura costituiscono il principale motivo di ostacolo alla scelta dello screening di massa dell'infezione da Hp. Vediamo perché:

a) Intanto, come detto sopra, l'efficacia del trattamento non è assoluta. Ciò pone in questi problemi soprattutto psicologici ed etici, perché in caso di insuccesso terapeutico ci troveremmo di fronte a persone alle quali dapprima abbiamo raccomandato il test in fase asintomatica, in funzione del rischio di cancro, e che poi si troverebbero impossibilitate a rimuovere proprio quel fattore di rischio. Nei confronti di queste persone avremmo quindi realizzato l'obiettivo opposto a quello prefissato, cioè un peggioramento dello stato di salute.

b) È possibile che le persone sottoposte a trattamento eradicante di Hp sviluppino un aumentato rischio di cancro dell'esofago. Per verificare questa inquietante ipotesi sono ancora in corso importanti studi, i cui risultati non sono previsti a brevissimo termine.

c) Ma soprattutto, attualmente su di un punto vi è unanime consenso internazionale: non c'è indicazione al trattamento eradicante nella persona asintomatica. Questa è di fatto la principale argomentazione che motiva la raccomandazione a non eseguire lo screening dell'infezione da Hp.

Si dispone di mezzi adeguati per la diagnosi? Esiste un test o un esame che permetta di rintracciare effettivamente la malattia?

Il test utilizzato è accettabile dalla popolazione?
Per poter definire i mezzi diagnostici adeguati ai fini di uno screening di massa, occorre valutare alcune caratteristiche fondamentali del test:

- la sensibilità, che è la principale caratteristica per un test di screening ed è in relazione alla capacità di rilevare effettivamente le persone affette, ovvero ad una bassa percentuale di falsi negativi;

- la specificità, che è in relazione ad una bassa percentuale di falsi positivi e che pure è importante negli screening (un'alta percentuale di falsi positivi comporta dispendio di esami di conferma e notevole disagio psicologico dei pazienti)

- il costo

- la facile riproducibilità

- l'accettabilità

I test diagnostici per Hp possono essere suddivisi in due gruppi: invasivi e non invasivi, questi ultimi basati sulla biopsia endoscopica della mucosa. La scelta del test da utilizzare dipende dalle esigenze cliniche $(9,10)$.

I test non invasivi comprendono l'Urea breath test, i test sierologici, la ricerca dell'Antigene nelle feci, la coprocoltura.

I test invasivi includono invece l'istologia, la coltura e la PCR su mucosa gastrica, il test rapido all'ureasi.

Non è scopo del presente lavoro considerare nel dettaglio le singole metodiche; basti qui ricordare il grado di sensibilità e di specificità degli esami, che in linea generale è oggi molto alto e quindi potenzialmente idoneo a finalità di screening. Per $i$ test più utilizzati, la sensibilità è pari almeno al $90-95 \%$, mentre la specificità arriva a sfiorare il $100 \%$.

Nel caso degli screening, ricordiamo brevemente che nella valutazione di un test dobbiamo correlare sensibilità e specificità ai valori predittivi positivo e negativo, i quali, in base al teorema di Bayes, sono connessi alla prevalenza della patologia nella popolazione. In breve, per una patologia poco diffusa nella popolazione, anche un test specifico avrà un potere predittivo positivo basso (molti falsi positivi) e viceversa. Comunque, anche questi parametri risulterebbero accettabili per effettuare lo screening.

Per quanto riguarda infine l'accettabilità dei test da parte di una popolazione sana, risulta evidente che $i$ test non invasivi avrebbero la preferenza da questo punto di vista e risulterebbero del tutto accettabili.

C'è evidenza di un vantaggioso rapporto costo/efficacia sia come guadagno di salute a lungo termine, sia di risorse risparmiate con la prevenzione? 
Da quanto visto in precedenza, discende che il rapporto costo/efficacia può forse essere favorevole nel sintomatico, ma certo non nell'asintomatico. In questo caso, è addirittura il rapporto rischio/beneficio a non essere inequivocabilmente favorevole; e di conseguenza, anche la valutazione della convenienza economica dell'intervento (oltretutto di costo non bassissimo) non potrà dare esito favorevole, né in termini di salute individuale, né in termini di salute pubblica.

Sono poi ancora in corso studi per valutare la mortalità correlata ad infezione da Hp (10); probabilmente nei prossimi anni si avranno ulteriori elementi per valutare con più precisione la domanda in questione.

La malattia può essere scoperta in fase di latenza $o$ all'inizio della fase clinica?

Sì, ma soltanto una piccola parte degli infetti evolve verso il cancro.

È ben conosciuta la storia naturale della malattia, specialmente nella sua evoluzione dalla fase di latenza alla fase sintomatica?

Da quanto detto si evince che la risposta è insoddisfacente, specialmente ai fini di una politica di screening.

\section{CONCLUSIONI}

Attualmente, pressoché tutte le Linee Guida più autorevoli e le raccomandazioni internazionali sono unanimi nel raccomandare di non eseguire lo screening dell'infezione da Hp nella popolazione generalizzata $(5,6,7,11,12,13)$. Molti documenti danno anche raccomandazione di non ricercare $\mathrm{Hp}$ nei soggetti con reflusso gastroesofageo, né nei soggetti con storia familiare di cancro gastrico o di ulcera peptica.

Le motivazioni di ciò, molteplici, risiedono non primariamente nella valutazione economica dell'intervento diagnostico e - quando indicato terapeutico, quanto nell'attuale non indicazione al trattamento dei soggetti asintomatici.

Le attuali raccomandazioni sono suscettibili di cambiamenti, in funzione delle conoscenze sulla storia naturale dell'infezione da Hp e sulla storia a lungo termine dei pazienti trattati con terapia eradicante.

\section{BIBLIOGRAFIA}

1. Wald, N. J. Guidance on terminology. J Medic Screen (1994) 1,76

2. Viano C.A., Vitelli A. Principi bioetici e screening, Le Scienze, (1987) 341, 76-79

3. Feldman RA. Epidemiologic observations and open questions about disease and infection caused by Helicobacter pylori. In: Achtman M, Suerbaum S, eds. Helicobacter pylori: molecular and cellular biology. Wymondham, United Kingdom: Horizon
Scientific Press, 2001:29-51.

4. Dixon MF. Pathology of gastritis and peptic ulceration. In: Mobley HLT, Mendz GL, Hazell SL, eds. Helicobacter pylori: physiology and genetics. Washington, D.C.: ASM Press, 2001:459-69.

5. European Helicobacter Pylori Study Group. Current Concepts in the Management of Helicobacter pylori Infection The Maastricht 2-2000 Consensus Report, 21-22 September 2000

6. Bazzoli F. Key points from the revised Maastricht Consensus Report: the impact on general practice. Eur J Gastroenterol Hepatol 2001;13:Suppl

7. CeVEAS-Centro per la Valutazione della Efficacia della Assistenza Sanitaria. Linea Guida Sulla Diagnostica dell' Helicobacter pylori.

Maggio 2000, rev. maggio 2001. http://web1.satcom.it/interage/ceveas $/ \mathrm{html} / \mathrm{doc} / 47 / \mathrm{LG} \operatorname{diagHP} 2002$. $\underline{\mathrm{PDF}}$

8. Uemura $\mathrm{N}$, Okamoto $\mathrm{S}$, Yamamoto $\mathrm{S}$, et al. Helicobacter pylori infection and the development of gastric cancer. N Engl J Med 2001;345:784-9.

9. Howden CW, Hunt RH. Guidelines for the management of Helicobacter pylori infection. Am J Gastroenterol 1998; 93:2330-8.

10. Suerbaum S., Michetti P. Helicobacter pylori infection, N Engl J Med (2002) 347, (15) 1175 - 86.

11. R Hunt, ABR Thomson. Canadian Helicobacter pylori Consensus Conference. Can J Gastroenterol 1998;12(1):31-41.

12. Howden C. W, Hunt R.H. Guidelines for the Management of Helicobacter pylori Infection. Am J Gastroenterol 1998;93:2330-8.

13. Passaro D. J., Chosy J. E., Parsonnet J. Helicobacter pylori: Consensus and Controversy. - Clin Infect Dis, Emerging Infections, 2002:35 (1 August), 298-304. 